\title{
Thermoacoustic Power Systems for Space Applications LA-UR-01-5811
}

\author{
Scott Backhaus ${ }^{1}$, Emanuel Tward ${ }^{2}$, and Michael Petach ${ }^{2}$ \\ ${ }^{1}$ Condensed Matter and Thermal Physics Group, Los Alamos National Laboratory, Los Alamos, NM 87545 \\ ${ }^{2}$ Space and Technology Division, TRW, One Space Park, Redondo Beach, CA 90278 \\ 1505-667-7545; backhaus@lanl.gov
}

\begin{abstract}
Future NASA deep-space missions will require radioisotope-powered electric generators that are just as reliable as current RTGs, but more efficient and of higher specific power $(\mathrm{W} / \mathrm{kg})$. Thermoacoustic engines can convert high-temperature heat into acoustic, or PV, power without moving parts at $30 \%$ efficiency. Consisting of only tubes and a few heat exchangers, these engines are low mass and promise to be highly reliable. Coupling a thermoacoustic engine to a low-mass, highly reliable and efficient linear alternator will create a heat-driven electric generator suitable for deep-space applications. Data will be presented on the first tests of a demonstration thermoacoustic engine designed for the 100-Watt power range.
\end{abstract}

\section{INTRODUCTION}

Thermoacoustic engines are a class of engines that convert high-temperature heat into acoustic, or PV, power without using moving parts. Recent innovations in thermoacoustics have allowed these engines to utilize the more efficient Stirling thermodynamic cycle (Backhaus, 2000). The result has been an increase in thermal-to-PV conversion efficiency from $\sim 21 \%$ to $30 \%$. The Thermoacoustic-Stirling Heat Engine (TASHE) that demonstrated $30 \%$ efficiency produced $\sim 1 \mathrm{~kW}$ of PV power.

Future NASA deep-space missions will require highly reliable (>100,000-hour lifetime), high specific power ( 10 W/kg), and efficient (13\%-25\%) thermal-to-electric converters in the 100$\mathrm{W}_{\mathrm{e}}$ power range (Mondt, 2001). These converters will be powered by high-temperature heat from the radioactive decay of ${ }^{238} \mathrm{Pu}$ contained in a General Purpose Heat Source (GPHS). Since a TASHE has no moving parts, it has the promise to be highly reliable, and it has demonstrated high efficiency in the $1-\mathrm{kW}$ power range. By coupling a TASHE to a linear alternator that takes its heritage from flight-proven, high-efficiency linear compressors used in space-based cryocoolers (Tward, 1999), a new type of reliable thermal-to-electric converter will be created.

The critical component in this converter is the TASHE. All past work on TASHEs has been done in the $1-\mathrm{kW}$ range and above. Scaling the TASHE down to the 100-W range, while maintaining its high efficiency, minimizing its mass, and providing easy thermal and mechanical interfaces to the rest of the power system, is critical to the success of this new conversion 
technology. The next two sections describe the design and construction of a 100-W class TASHE and its initial tests using an acoustical resonator as a replacement for a linear alternator.

\section{APPARATUS}

The layout of the TASHE is shown in Figure 1. Essentially, it is composed of a looped flow path filled with high-pressure helium gas. The loop contains the regenerator, heat exchangers, and other ductwork necessary to force the gas in the regenerator to execute the Stirling cycle (Backhaus, 2000). The mass of the first laboratory TASHE up to the resonator/alternator interface is approximately 900 grams. The total mass was measured after several pieces of instrumentation were installed, but before a final closure weld was made. At this $100-\mathrm{W}$ scale, the TASHE contributes less than $10 \%$ of the mass in the $10 \mathrm{~W} / \mathrm{kg}$ space power system desired by NASA (Mondt, 2001). Additional mass savings are expected in a flight design. If designed for a flight system instead of laboratory convenience, much of the instrumentation and bolting flanges would be removed. Also, much of the extraneous metal in the TASHE could be removed, and aluminum could be substituted for several of the stainless steel components.

In Figure 1, the oscillating flow through the lower face of the secondary ambient heat exchanger impinges on a piston of a linear alternator. Our initial tests use an acoustical resonator that mimics the linear alternator (not shown). The reactive impedance of the resonator behaves like the mass of the piston, and the dissipation in the resonator and an adjustable acoustic load (Backhaus, 2000) duplicates the electrical load across the linear alternator's electrical terminals.

The oscillating flow path in the top of the loop is a small gap between two Inconel 625 parallel plates forming the hot heat exchanger. High-temperature heat enters the system by solid conduction through the top plate (maximum operating metal temperature of $650{ }^{\circ} \mathrm{C}$ ). There are many small-diameter pins that are machined into the bottom plate and welded into holes through the top plate. These do not serve to increase the heat transfer area. They are supports to keep the thin top plate from bowing outward under the action of the internal pressure. In the test, the high-temperature heat is generated by 4 electrically-powered heaters embedded in a nickel block (not shown) cemented to the top surface of the hot heat exchanger.

Since the TASHE has no moving parts, its geometry can be very flexible. The design shown in Figure 1 is chosen so that the hot end of the TASHE is flat and exposed on one end. This configuration allows for a simple thermal and mechanical interface to the GPHS. In principle, solid conduction from the GPHS to the hot heat exchanger could be used to couple the heat into the TASHE. An interface of this type should not require any additional metal to transport the heat to the TASHE or to support the GPHS. This provides an additional mass savings when the converter is integrated with the rest of the power system. With no moving parts in the hot end of the TASHE, creep of the hot end material induced by the high temperatures and high internal pressure will not cause any performance issues due to the loss of a critical tolerance.

Proceeding down the right leg of the loop is the regenerator, which is composed of a stack of stainless-steel screen housed in an Inconel 625 cylinder. Below the regenerator is the main ambient heat exchanger. It is an aluminum cylinder with 48 long holes drilled through. In these 
laboratory tests, the waste heat rejected by the TASHE through this heat exchanger is finally carried away by cooling water flowing through several turns of copper tube wrapped around the outside of the heat exchanger.

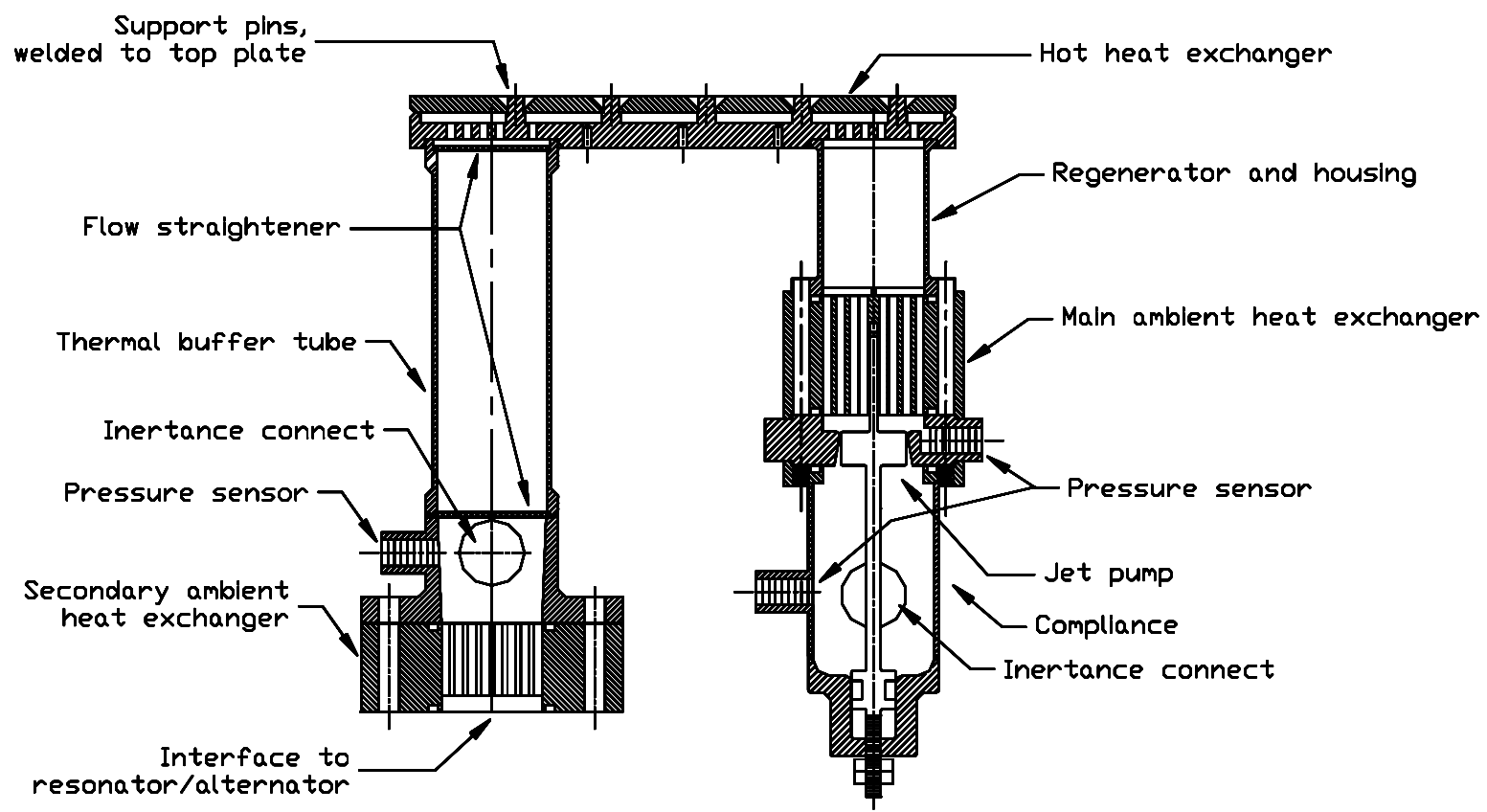

FIGURE 1. Cross Section of the TASHE. All parts have circular symmetry except for the hot heat exchanger. The two circles labeled "Inertance connect" are the locations where a long tube is welded to complete the loop around the TASHE. The linear alternator/resonator is not shown. The overall height of the TASHE is approximately $15 \mathrm{~cm}$.

In the left leg of the loop below the hot heat exchanger is the Inconel 625 thermal buffer tube (TBT). The TBT allows acoustic power to flow away from the hot heat exchanger while thermally isolating the hot end from ambient temperature. To avoid large convective heat leaks, it is crucial that the flow in the TBT remain thermally stratified. Any jetting out of the adjacent heat exchangers would cause mixing of hot and cold gas. Therefore, flow straighteners are placed at either end of the TBT. Below the TBT are a T-junction and a secondary ambient heat exchanger. This heat exchanger restricts the thermal gradient to the TBT protecting the resonator, and later the linear alternator, from the high temperatures of the hot heat exchanger. Except for its length, it is identical to the main ambient heat exchanger. In future designs, the main and secondary heat exchangers, as well as some of the components below the main heat exchanger, may be machined into a single aluminum block. This will simplify the thermal and mechanical interface at the ambient end of the TASHE.

The heat exchangers and TBT described above form the thermodynamic section of the TASHE. A crucial acoustic network is formed by the open volume, or compliance, below the main ambient heat exchanger and the inertance tube that connects the ambient end of the TBT to the compliance. This network generates pressure and velocity oscillations in the regenerator that drive the gas through the Stirling cycle (Backhaus, 2000). The inertance tube is connected to the penetrations shown in the compliance and just above the secondary ambient heat exchanger. As 
the gas oscillates back and forth through the inertance, the compliance is pressurized and depressurized generating pressure oscillations in the regenerator. Also, the gas in the inertance must accelerate during the oscillations. The inertia of the gas creates an oscillating pressure drop across the inertance. This pressure drop also appears across the regenerator, and it drives the oscillating velocity in the regenerator. The operation of the network can loosely be compared to that of a displacer piston in a free-piston Stirling engine. A detailed description of how this network operates can be found elsewhere (Backhaus, 2000).

The final component in the loop is located between the main ambient heat exchanger and the compliance. Called a "jet pump", it is an annular diffuser with the small end facing the main ambient heat exchanger. The area of the small-end opening is adjustable by moving the central plug in the axial direction. The asymmetry of the flow out of the small end creates an adjustable time-averaged pressure drop that generates a steady flow around the TASHE's loop. This flow is used to cancel a parasitic steady flow around the loop generated by acoustic streaming. Without the action of the jet pump, the parasitic streaming flow would cause a convective heat leak on the hot heat exchanger lowering the efficiency of the TASHE. A detailed description of this phenomenon can be found elsewhere (Backhaus, 2000).

The TASHE is instrumented with pressure sensors and type-K thermocouples. The pressure sensors are located in the compliance, between the jet pump and cold heat exchanger, and at the junction between the inertance and TBT. These sensors measure the pressure drop across three key elements in the TASHE: the jet pump, regenerator, and inertance. If these pressure drops agree with numerical calculations, the actual oscillating velocities in the TASHE are assumed to be close to the calculated velocities. An additional set of pressure sensors are placed in the resonator and variable acoustic load. These measure the amount of acoustic power flowing into the resonator, the same power that impinges on the face of the linear alternator's piston. A description of this measurement technique can be found elsewhere (Backhaus, 2000 and Fusco, 1992).

Thermocouples are placed in several locations around the loop. Three are inserted into tightfitting pockets drilled into the regenerator itself; one near the ambient end, one near the hot end, and a third at the axial midpoint. These thermocouples are used to determine what jet pump setting suppresses the parasitic steady flow around the TASHE's loop (Backhaus, 2000). When the parasitic flow is stopped, the temperature profile in the regenerator is nearly linear. Five thermocouples spot welded to the outside of the TBT serve a similar purpose. Any net steady flow around the loop will affect the temperature profile in the TBT. Also, acoustic streaming cells confined to the TBT (Olson, 1997) will modify its temperature profile. This second type of streaming will also cause a heat leak on the hot heat exchanger lowering the efficiency of the TASHE. The measurement of the temperature profile in the TBT helps to determine if these effects are significant. To analyze the effectiveness of the hot heat exchanger, there are eight thermocouples touching the outer surface or embedded in the metal plates. Five are distributed evenly along the centerline of the plates between the regenerator and TBT. Two are at either edge of the plates halfway between the regenerator and TBT. An additional thermocouple is embedded in the center of the lower plate. Finally, there is a thermocouple placed at the OD of the main ambient heat exchanger. It is used to analyze the effectiveness of this heat exchanger. 


\section{MEASUREMENTS}

To make rapid progress, the TASHE is tested on an acoustical resonator that mimics the both the reactive and resistive properties of a linear alternator attached to an electrical load. However, the resonator is lossy enough that a single TASHE would only be able to drive it at or near the maximum allowable temperature applied to the hot heat exchanger metal. To avoid this difficulty, two identical TASHEs have been built and both are attached to the same resonator using a $30^{\circ} \mathrm{Y}$-junction bored into a single brass block. The data presented below is taken from one of the two TASHEs. The data for the second is quantitatively similar.

\section{Temperature Profiles}

Figure 2 shows the temperature distribution in the regenerator and TBT as well as the temperature of the hot and cold heat exchanger metal. As found previously, the TASHE delivers the most power to the resonator when the jet pump small-end area is adjusted so that the three measured temperatures in the regenerator fall along a straight line (Backhaus, 2000). In this condition, the jet pump is generating the amount of steady flow necessary to cancel the parasitic streaming flow and eliminate any potential convective heat leaks on the hot heat exchanger. Numerical calculations (Ward, 1994) confirm that when there is no net steady flow around the loop, the axial temperature profile of the regenerator is nearly linear.

If there is no boundary-layer streaming or any other mixing processes occurring in the TBT (Olson, 1997), numerical calculations indicate that its temperature distribution should be nearly linear as well. The three TBT profiles shown in Figure 2 show small deviations from linear. These could be due to a small amount of boundary-layer streaming, inadequate flow straightening at the TBT ends, or possibly a radiation heat leak from the hot heat exchanger. However, at this early stage of testing, it is apparent that the TBT is performing nearly as expected, and these deviations from the numerical predictions are small.

In the hot heat exchanger section of Figure 2, the open symbols connected by lines are the measured temperatures on the top surface of the heat exchanger along the axis connecting the centerlines of the regenerator and TBT. For the data at the highest temperature, approximately 80 Watts of heat is transferred through the top plate. At these power levels, the temperature along this axis is fairly constant showing an $\sim 20{ }^{\circ} \mathrm{C}$ variation from the midpoint to the location over the regenerator centerline. At higher power levels, this variation will increase due to increased heat transfer, but the oscillating gas displacement will increase as well. The second effect will tend to reduce the temperature variation. Two additional thermocouples are placed at the transverse edges of the heat exchangers halfway between the regenerator and TBT. The crosses in Figure 2 indicate their average temperature. These data show that there is very little temperature variation in the direction transverse to the oscillating flow. This provides evidence that the oscillating flow is accessing all of the surface area in the hot heat exchanger even though the diameter of the regenerator and TBT is smaller than the width of the heat exchanger. With the flow accessing the entire surface exposed to the heat source, concerns about hot spots and associated pressure vessel failures are reduced. The final open symbol, which is not connected by a line, is the temperature at the center of the bottom plate. With very little heat transfer 
occurring between the oscillating gas and the bottom plate, this temperature is very close to the gas temperature.

A straight line fit to the three temperatures in the regenerator can be used to extrapolate the gas temperature at the hot and ambient ends of the regenerator. The extrapolated gas temperature at the ambient end is approximately $5{ }^{\circ} \mathrm{C}$ higher than the metal temperature at the OD of the main ambient heat exchanger. This gas-metal temperature defect is inline with expectations at these power levels. At the highest hot-end temperature, the extrapolated hot-end temperature is approximately $50{ }^{\circ} \mathrm{C}$ cooler than the hot heat exchanger top plate. The measured temperature defect is significantly higher than expected at these power levels.

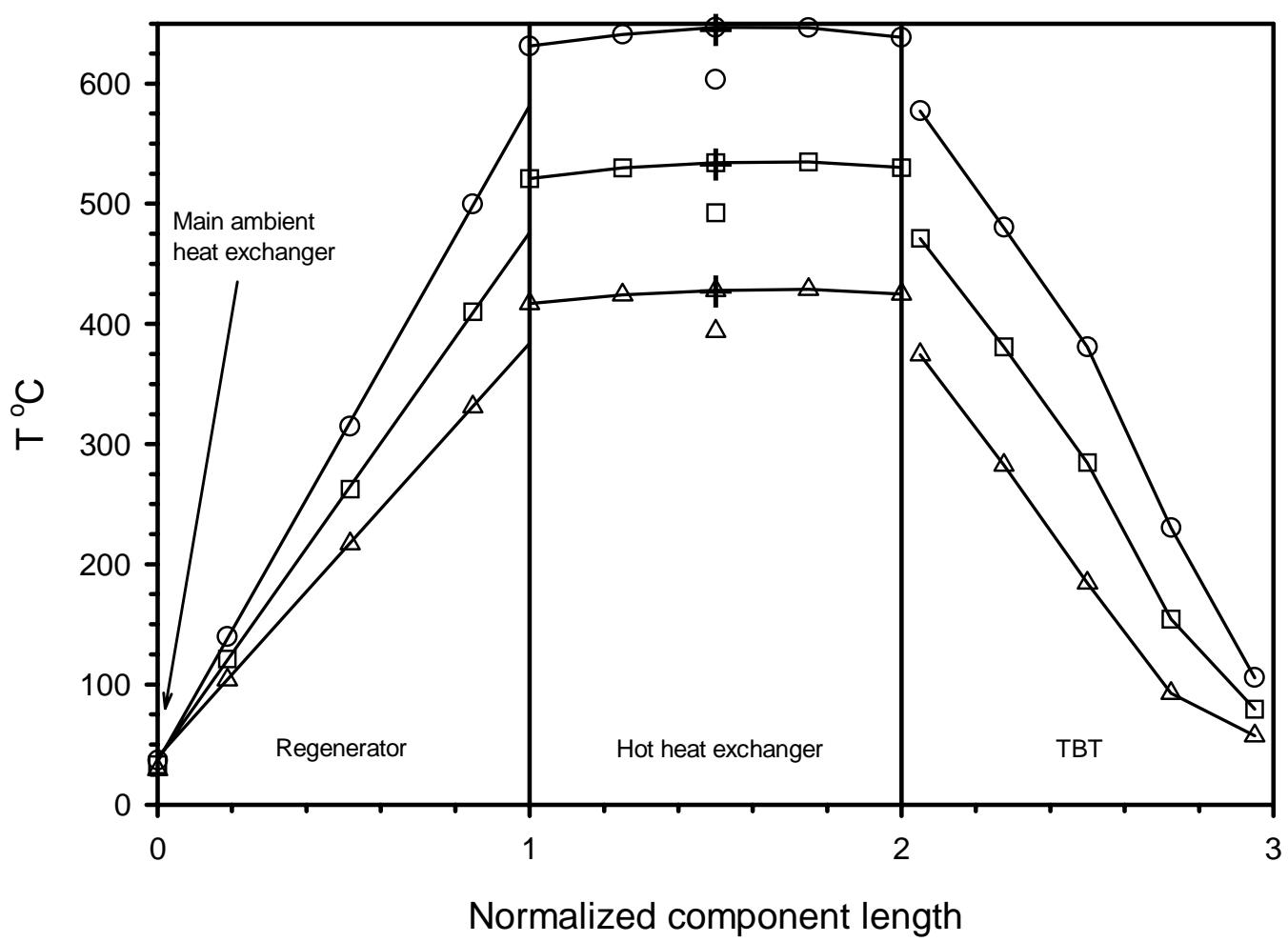

FIGURE 2: Temperature Distribution Through the TASHE. The open circles, squares, and triangles are the measured temperatures at three different hot-end temperatures at an oscillating pressure amplitude of approximately $150 \mathrm{kPa}(\sim 25 \%$ of design amplitude). The jet pump has been adjusted to ensure a linear temperature distribution in the regenerator. The open symbol in the hot heat exchanger section not connected by a line is the temperature of the hot heat exchanger bottom plate. The three crosses are the temperature at the edges of the heat exchanger plates for the different hot end temperatures. The straight lines in the regenerator section are least-squares fits to the 3 data points in that section. Although each section of the TASHE represented above is of different length, each length has been normalized to 1 to aid in the presentation of the data. 
Part of this difference may be due to the positioning of the thermocouples. Only the very end of the thermocouple sheath actually touches the top plate. The location of the thermocouple junction, which is slightly removed from the end of the sheath, may be positioned in the cement which bonds the nickel heater block to the top plate. This would lead to an erroneously high temperature reading. In future tests, steps will be taken to ensure the thermocouples are reading the temperature of the top plate.

\section{Pressure Drops}

With the temperature distributions around the TASHE loop reasonably well understood, the pressure drops across the various components are measured. Figure 3 shows the measured and numerically calculated pressure drop across the regenerator, jet pump, and inertance at an acoustic pressure amplitude of $150 \mathrm{kPa}$. There is reasonable agreement between the measured and calculated total pressure drop across the inertance. This gives confidence that the numerical calculation is accurately predicting the oscillating velocity throughout the TASHE. The main discrepancy is in the pressure drop across the jet pump. This may be due to an alternate sensor mounting technique used to accommodate the small size of the TASHE. Modifications to the mounting will be made for future tests. However, the agreement of the total pressure drop with predictions is adequate for this early stage of testing.

\section{CONCLUSIONS}

In this initial testing, the TASHE has performed as expected. The temperature distributions in the heat exchangers and other components are in agreement with estimates. The oscillating pressure distributions show reasonable agreement as well. Higher power tests will be performed on an acoustical resonator before integrating the TASHE with a linear alternator to form a thermal-to-electric power converter. The flexibility afforded by not having moving parts has already shown some advantage. The thermal and mechanical interface to the heat source is a simple flat plate whose entire surface is cooled by the oscillating gas. This reduces concerns about hot spots at the heat-source interface. Finally, at this 100-W scale, the TASHE contributes less than $10 \%$ of the mass in the $10 \mathrm{~W} / \mathrm{kg}$ space power system desired by NASA (Mondt, 2001)

\section{ACKNOWLEDGMENTS}

The authors would like to thank D. L. Gardner, C. Espinoza, and R. Haggart for their technical assistance in the construction of the TASHE. This work is funded by NASA under contract number NAS3-01103. 


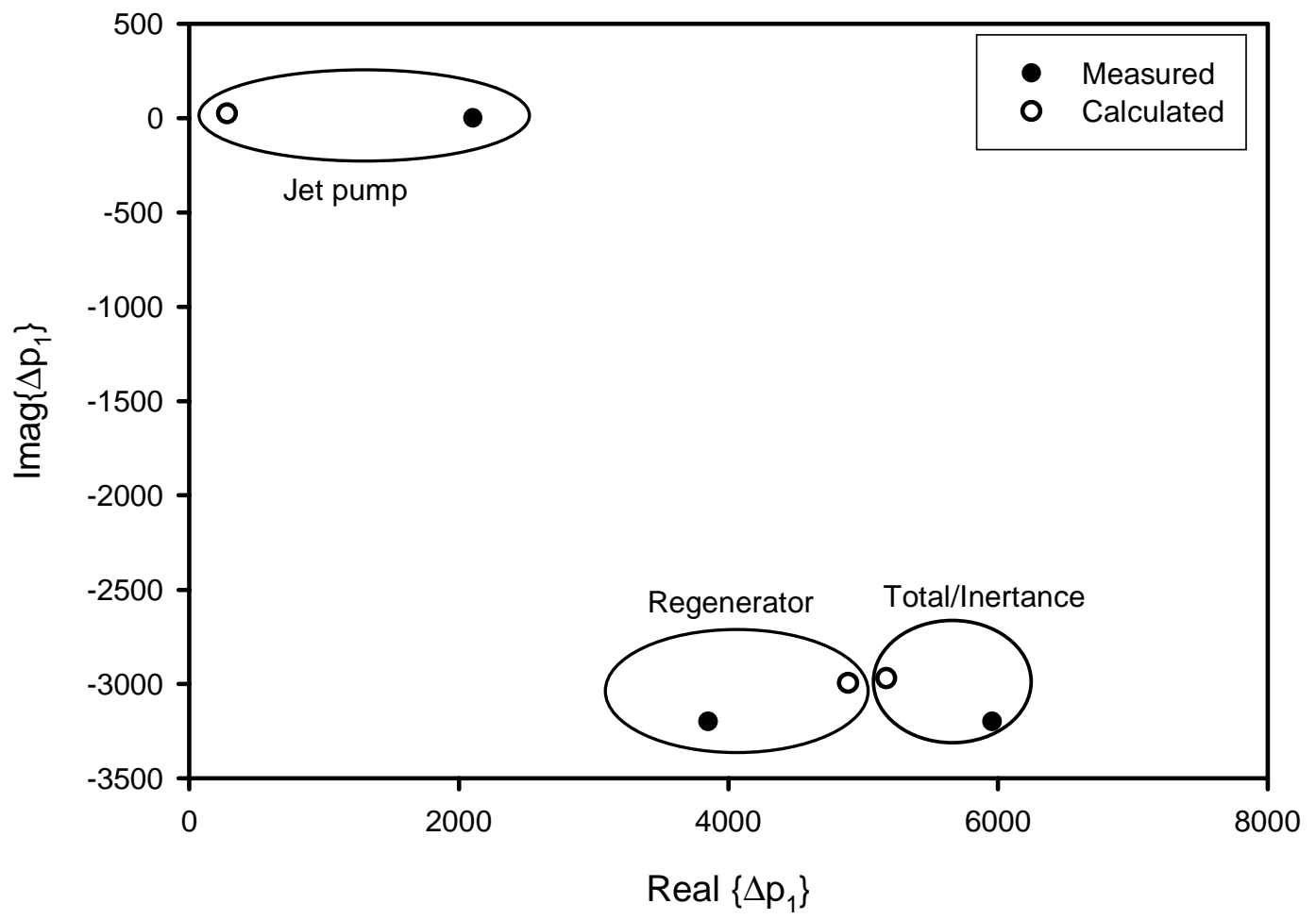

FIGURE 3: Acoustic Pressure Drops. Measured and calculated pressure drops across the regenerator, jet pump, and total drop across the inertance tube at an oscillating pressure amplitude of $150 \mathrm{kPa}$ ( $\sim 25 \%$ of design amplitude).

\section{REFERENCES}

Backhaus, S., and Swift, G. W., "A Thermoacoustic-Stirling Heat Engine: Detailed Study,” Journal of the Acoustical Society of America, 107, 3148-3166, 2000.

Fusco, A. M.; Ward, W. C.; and Swift, G. W., "Two-Sensor Power Measurements in Lossy Ducts," Journal of the Acoustical Society of America, 91, 2229-2235, 1992.

Mondt, J. F., "Advanced Radioisotope Power System Technology Developments for NASA Missions 2001 and Beyond," Proceedings of the IECEC, 133-139, Savannah, GA, August, 2001.

Olson, J. R., and Swift, G. W., "Acoustic Streaming in Pulse Tube Refrigerators: Tapered Pulse Tubes,” Journal of the Acoustical Society of America, 37, 769-776, 1997.

Tward, E., and Davis, T., "High Efficiency Cryocooler," AIAA, \#99-4564, Albuquerque, NM, Sept. 1999.

Ward, W. C., and Swift, G. W., "Design Environment for Low Amplitude Thermoacoustic Engines, DeltaE," Journal of the Acoustical Society of America, 95, 3671-3672, 1994. Fully tested software and users guide available from Energy Science and Technology Software Center, US Department of Energy, Oak Ridge, Tennessee. To review DeltaE's capabilities, visit the Los Alamos thermoacoustics web site at http://www.lanl.gov/projects/thermoacoustics/. For a beta-test version, contact ww@lanl.gov (Bill Ward) via Internet. 